\title{
A DIDACTIC ITINERARY THROUGH MATHEMATICS, SCIENCE AND ART ON ESCHER'S PERIODIC DRAWINGS
}

\author{
Simone Canciani \\ High school of Art, Italy
}

\begin{abstract}
The purpose of this analysis is to show that strong connections might exist between the teaching of mathematics, science and art. This is due to the particular geometrical configurations behind several paintings and other artistic creations. Therefore, the knowledge of this connection might be of help for the students to guess that mathematics and science also have possible applications to art. This is particularly relevant for the high schools of art. Since too often, mathematics is taught in high schools without any relevant link to reality, this can be one of the reasons of students'disaffection for this subject. In this analysis, a teaching experiment developed by the author in an Italian (Udine) high school of art is proposed. In particular, Escher's periodic drawings, and the ornamental motifs that characterize many products in reality, offer an interesting opportunity to make high school students understand the relevance of mathematics and especially of geometry in art. The discovery of mathematical laws, that derive from crystallography, behind Escher's drawings should persuade students that such an artistry is reachable only by means of mathematical knowledge. In what follows the main features of the outlined didactical proposal are presented and discussed.
\end{abstract}

Keywords: Escher, periodic drawings, mathematics and art, mathematics teaching, cristallography

\section{Introduction}

Many learners attending high schools of art consider mathematics a difficult and useless subject. Therefore, some strategy is to be found to improve their attitude towards this discipline. One possibility is to link mathematics to students' artistic interests. Another one is to show how mathematics is involved in reality, for example, in nature.

Following this idea, natural science offers many starting points to discover the beauty and symmetry of the world. Some shapes in nature, like crystals, are a fascinating example which shows how and why the concept of symmetry is relevant (Weyl, 1952).

Escher's work on periodic drawings constitutes a bridge through art, mathematics and natural science (Castaldi \& Ghione, 2011) since he used geometry and the properties of the crystal structure in an artistic way. That's why a didactic itinerary based on Escher's periodic drawings could provide students with the necessary motivation to deal with mathematical concepts.

The first question which can be posed concerns the relation between Escher's drawings and a remarkable mathematical problem regarding planar tessellations, that is, which forms can be used to tile a plane. After defining the concept of tessellation and particularly those of regular and uniform tessellation, the underlying structure of Escher's compositions is investigated, without considering the tile's shape. That is because the problem of tessellation is solved only for certain types of polygons and is, in general, a very complex matter that cannot be easily approached in high schools.

However, related to this problem, there is another general issue that can actually be approached in a didactical way and that, in some cases, can explain the origin of some tessellations, particularly many of Escher's. This analysis combines Escher's periodic drawings and Hilbert's regular point patterns. 
The majority of this work outlines a didactical experimentation carried out in an Italian "Liceo Artistico", in an attempt to align the study of mathematics, and in particular, of geometry, with the arts.

The purpose of this activity was twofold: first, to have students analyse some of Escher's drawings and be able to understand the underlying "secrets" behind his compositions; second, to enable students to apply the techniques of geometry in order to create periodic drawings themselves, solely using isometries to completely fill a plane.

This didactic proposal should be particularly significant for a number of reasons: firstly, because it offers students a different perspective on mathematics. This new perspective, when compared to the traditional approach, can help low-engagement students to regain interest in the subject and improve their results. Secondly, the theme of periodic drawings has a relevant practical application in art: consider iron grating, perimeter walls, or printings on cloth - very often these present symmetrical ornamental motifs. Thirdly, there is not a single curriculum in a "Liceo Artistico" that does not include the study of periodic drawings on some level, from graphics to figurative arts, from fashion design to architecture. Finally, for students that will continue scientific studies, periodic drawings will help them see what they will one day find in fields such as (among many others) abstract algebra (Morandi, 2003) or crystallography (Rigault, 1976).

The main aim of this work is to provide mathematics teachers with some useful material on the periodic drawings subject. Insofar as high schools of art are concerned, this didactic itinerary presents a contribution to define a more suitable mathematics curriculum for students.

\section{Methodological Approach}

The activity was carried out in the first period of the 2019-2020 school year, and it consisted of 4 meetings of 2 hours each. It was one of the activities which are part of the PLS ("Progetto Lauree scientifiche", namely "Scientific Degree Project"), an Italian national programme whose aim is, among the others, to involve high school learners in the scientific culture.

The participation was voluntary; 17 students from different classes were involved. Some teachers participated voluntarily in the meetings, as well. They were mathematics teachers, fashion design teachers and figurative art teachers.

The learners were guided to the comprehension of the concepts by some theoretical lessons alternated with practical activities. More or less, 4 hours were dedicated to practical exercises. The attendants were provided with instruments and material (white and transparent paper sheets, scissors, glue, pencil, ruler, rubber) so that they could test personally the possible applications of theoretical results.

The teacher had the role of supporting the students in their activities. 


\section{Figure 1}

A Lace Pattern

\section{Planar Tessellations}

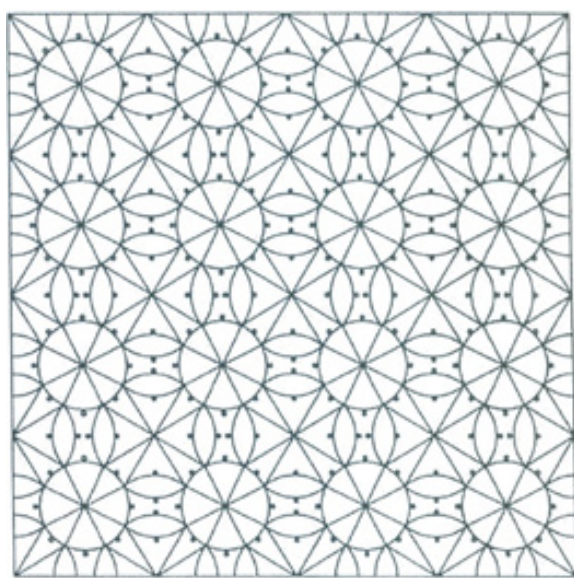

Starting with Figure 2: how could Escher realize this composition? This is a question that would normally arise when visiting an exhibition of the Danish artist, and a question that usually gives the impression that the author's art could not be grasped by just anyone. However, we will see that, in fact, it is possible at least to comprehend the methods used by Escher, even though Escher's artistry and imagination might remain out of reach for most people.

\section{Figure 2}

Escher's Fishes

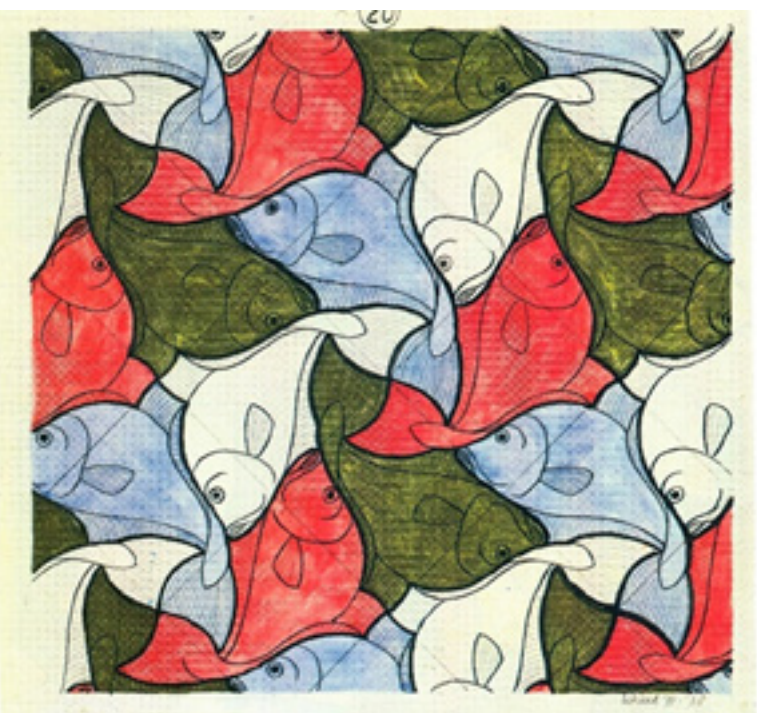


So, what is a planar tessellation? In general, it is a collection of polygons that fill a plane and intersect each other, at most, in one side or vertex (Dedò, 1999, p. 32). We will see that we can create tessellations that are not made up of polygons, but rather of shapes obtained by the deformation of the sides of the polygons (as in Escher's fishes).

Following this definition, we can ask ourselves some questions. For example: which convex polygons can tile the plane? In which way can different polygons be used for a tessellation? Is there a limit for the number of sides of a convex polygon to be used as a tile?

As a proof of the fact that this topic is of actual interest, we can consider that it could be demonstrated that, if $\mathrm{P}$ is a convex polygon with 7 or more sides, then $\mathrm{P}$ cannot tile the plane. Whereas for hexagons, the problem is solved, as we know the typologies of hexagons that can tile the plane, for pentagons, the problem is still unsolved! (Dedò, 1999, p. 59)

\section{Regular Tessellations}

Among tessellations, the first ones that are suitable for a systematic analysis are regular tessellations, i.e. those made up by regular polygons. It's not difficult to realize that the only regular tessellations are those made up by squares (Figure 3), equilateral triangles (Figure 4) and hexagons (Figure 5).

\section{Figure 3}

A Square Tessellation

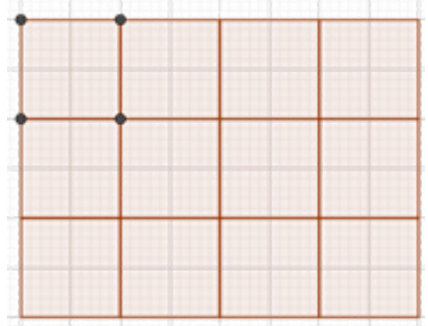

\section{Figure 4}

A Triangular Tessellation

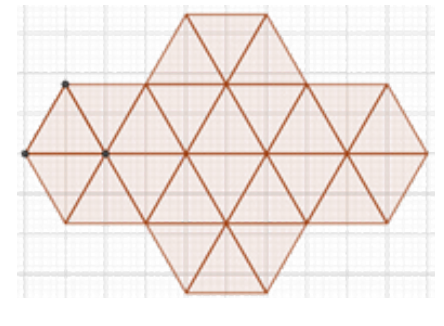

Figure 5

A Hexagonal Tessellation

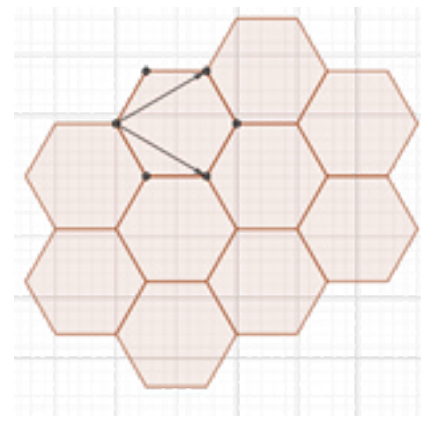

Exercise: why can only these polygons tile the plane?

In addition to regular tessellations there are uniform tessellations (those made up by regular but different polygons), whose properties were investigated by Kepler in his Harmonice Mundi, of which I provide some figures below (Aiton et al., 1997, p 104): 


\section{Figure 6}

Kepler's Uniform Tessellations from Harmonice Mundi
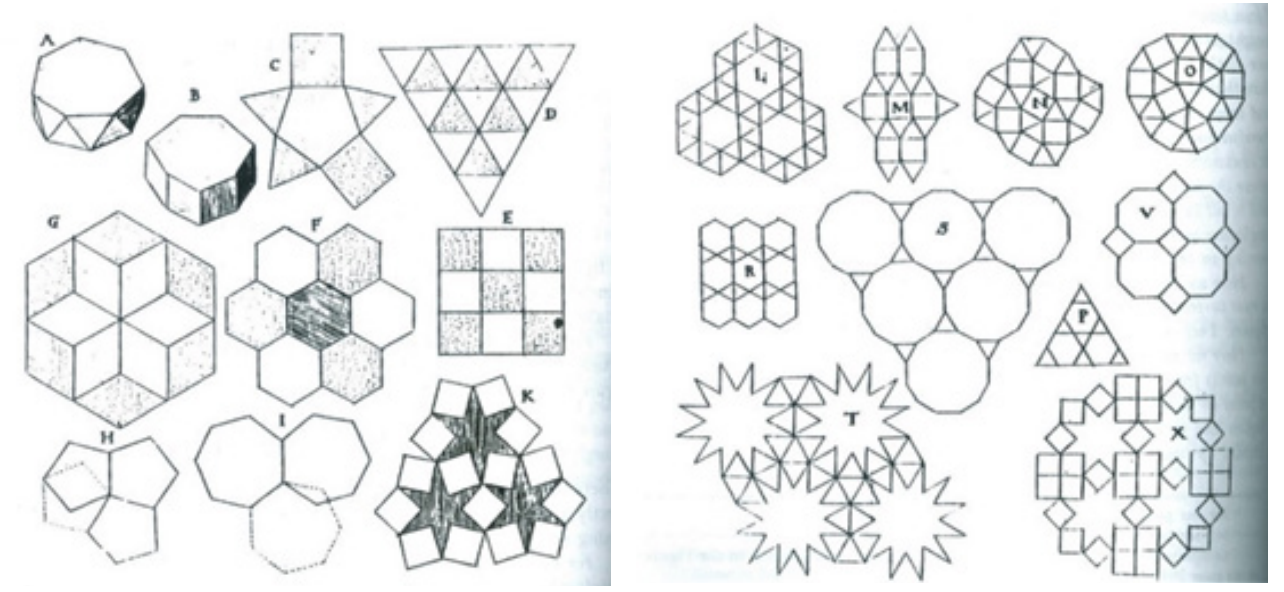

Regular tessellations have also been extensively studied and classified (Dedò, 1999, p. 46), but they always concern polygons. "Free" shapes, such as Escher's fishes, aren't included in these cases.

How can we proceed, then? It is probably necessary to change the point of view.

\section{Regular System of Points}

As we look at the fishes' drawing (Figure 2), we discover that the main characteristic of such a composition is the total lack of a reference point. Every object (fish) has the same position in relation to the others. Such an arrangement of objects is called a regular system (Hilbert, 1952, p. 32). In order to obtain shapes that can tile the plain, it is useful to examine what are all the possible regular systems of points.

Figure 7

A Regular System of Points

\section{Figure 8}

The Two Sets of Segments are Superimposable
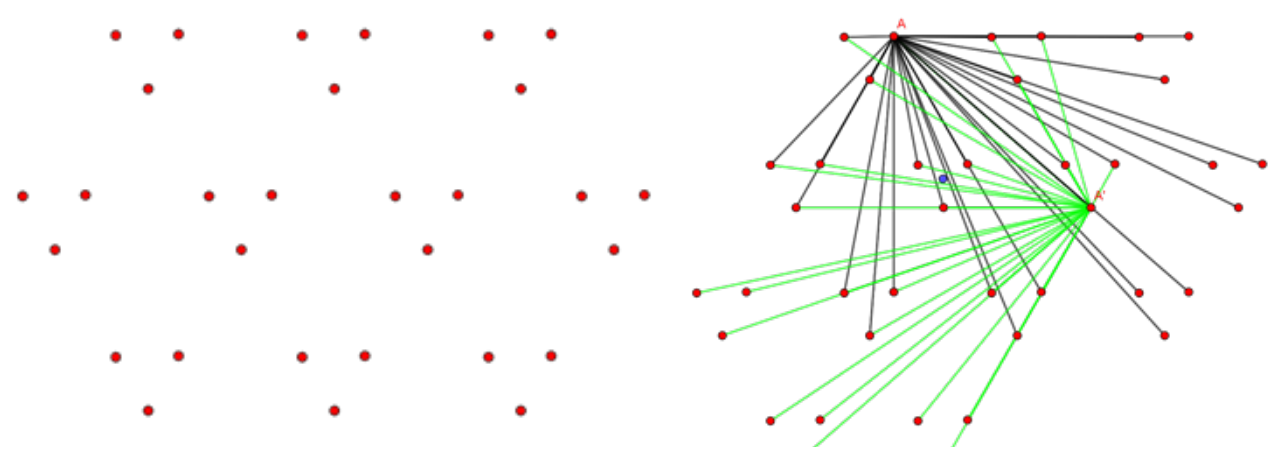
In Figure 7 a regular system of points is shown. None of the points drawn have a different position in relation to the others. This concept can be visually explained as in Figure 8: starting from a point A, we draw all the segments with one endpoint in A and the other in any other point of the regular system. Then, the same construction is developed starting from another point A'. So, there are two sets of segments that are superimposable; i.e., there exists an isometry that brings the first set of segments onto the second one (in the case shown in Figure 8, the isometry is the rotation of $120^{\circ}$ around the blue point).

Without drawing all the possible segments, this property of a regular system of points can be expressed by assuming that every point could be moved to any other by an isometric transformation of the system in itself.

Now it is explained how a regular system of points can be created. The essential idea is the following, and it is based on the mathematical concept of a group. The concept of a group, that normally is not part of teaching programs in high schools, is particularly useful here. It could be the right opportunity to introduce it. Otherwise it could be introduced earlier, for example dealing with the (finite) group of rotations that fix a regular polygon.

Seeing that, given two transformations of the system in itself, their composition is still a transformation of the system in itself; moreover, since for every transformation, there always exists an inverse transformation, then the set of all transformations of a system in itself is a group. So, a regular system of points is built by starting at an arbitrary point and applying a certain isometry group to that point in order to create infinite other points.

These isometries must also include translations along two non-parallel directions, as we consider periodic patterns, that repeat themselves ad infinitum, filling the whole plane. The other possible transformations, like rotations or reflections, have to be compatible with the translations in the group (which are always present).

Therefore, the following step is to seek to discover all the distinct groups of isometries containing two non-parallel translations. These groups are usually named planar crystallographic groups, and it can be demonstrated that there are only 17 (An algebraic demonstration of this fact can be found in Morandi, 2003).

The following exercise is conceived to allow students to comprehend the underlying structure of Escher's drawings; by following the exercise, they should be able to understand the relation between the Artist's drawings and the regular system of points.

\section{Figure 9}

\section{The Underlying Structure of Escher's Fishes}

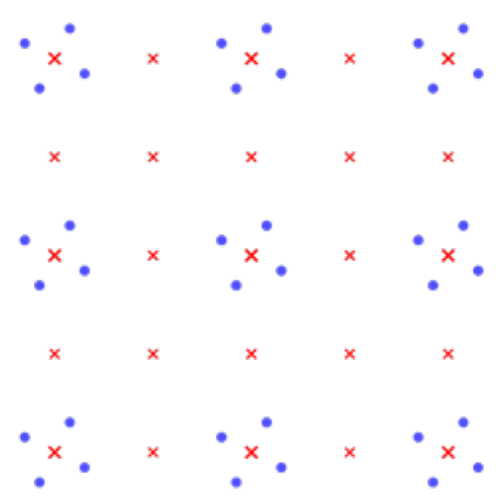


Exercise: find out all the points in the same position in Escher's fish drawing in Figure 2 (for example, each eye) and mark them on a transparent paper sheet. Also try to look for all the centres of rotation in the composition. Mark them in a different way (for example with an " $x$ "). Remove the transparent paper sheet and verify that you obtain an image similar to the one shown here on the side (Figure 9). On a white paper sheet, copy the structure that you have on the transparent sheet and verify that, through suitable rotations, you can make the system correspond with itself.

The $\mathrm{p} 1$ group (only with translations)

The simplest group is the one where isometries are only translations (along two different directions, at least): in this case we obtain a lattice as in Figure 10 (The p1 notation comes from crystallography as in Rigault, 1976).

Figure 10

A Lattice Generated by Two Non-parallel Translations

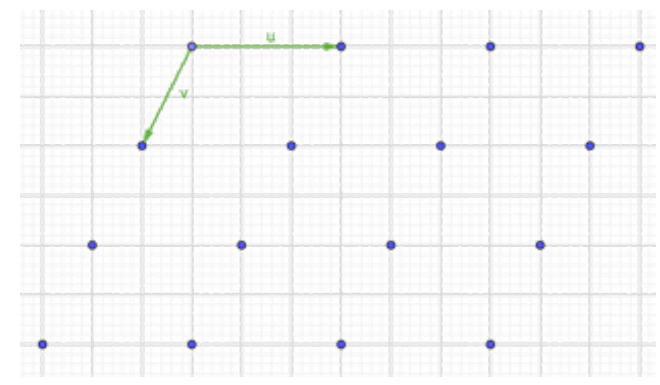

\section{Figure 11}

A Tessellation Obtained by the Modification of the Sides of the Parallelogram

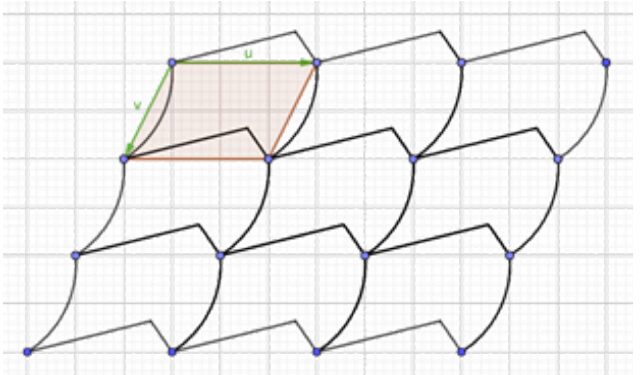

The group generated by the two translations $u$ and $v$ contains only translations and these will be, in this case, the only admissible isometries. The parallelogram limited by the two minimal translation vectors is called the basic parallelogram. It can completely fill the plane by action of the translations. Moreover, inside the basic parallelogram there aren't equivalent points, i.e. points that correspond to each other by means of some group action. For this reason, it is also called the minimal figure (The minimal figure is called "unit cell" in Hilbert, 1952, p. 63).

Once we have found the minimal figure, we can draw some ornamental motif inside, otherwise we can modify its perimeter, thus obtaining a plain tessellation (Figure 11) whose tile is less ordinary than the starting parallelogram. Here, in fact, we can see that the deformations on the two non-parallel sides are "carried on" by the $u$ and $v$ vectors and by their combinations. From this it is clear, that the new tile will have the same area of the minimal figure.

In spite of its simplicity, this idea can lead to remarkable artistic results, as you can see in Escher's Pegasus drawing (Figure 12). 


\section{Figure 12}

Escher's Pegasus

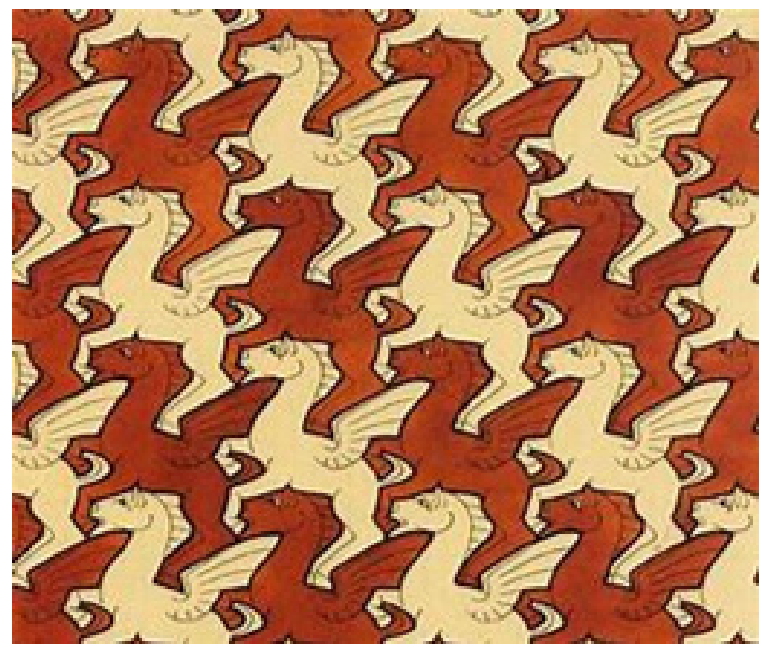

Exercise: starting from Escher's Pegasus, find the basic parallelogram and the minimal translation vectors. In which way has the basic parallelogram been deformed?

Rotations

While the simplest case is one in which only translations are present, let us investigate what happens if we introduce a $180^{\circ}$ centre of rotation. We call it a 2 -fold centre of rotation. To introduce the argument, two lemmas are presented (Hilbert, 1952, p. 63):

1. If, in a symmetry group, there is a rotation of an angle about a point $A$ and a translation vector $u$, then all the points obtained by the action of translations on $A$ are centres of rotation (of the same angle ).

2. If, in a symmetry group, there is a rotation of an angle about a point $A$ and a translation vector $u$, then there is necessarily a translation vector $u$, formed by the rotation of $u$ of the same angle.

Proof of the first lemma (Figure 13).

\section{Figure 13}

Generation of Centres of Rotation

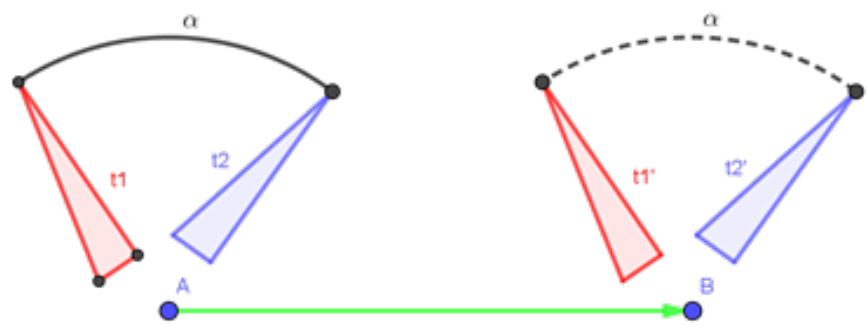


Let $A$ be a centre of rotation of an angle $\alpha$ and $u$ a translation vector. The triangle $t 1$ is translated in $t 1^{\prime}, t 1$ is rotated in $t 2$ and $t 2$ is translated in $t 2^{\prime}$.

\section{Figure 14}

Generation of Translation Vectors

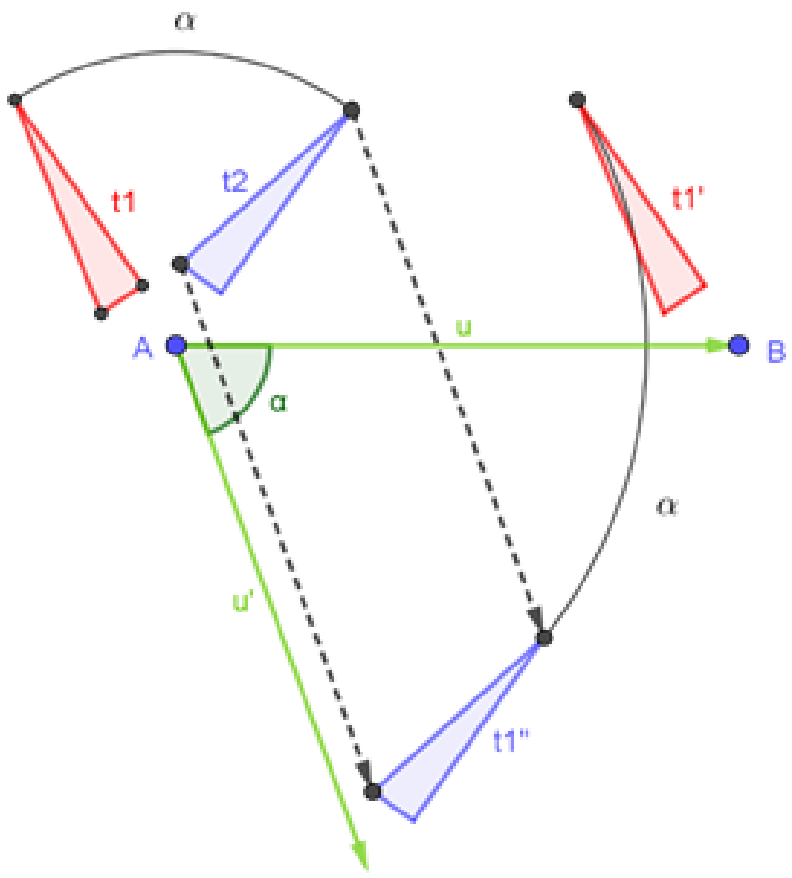

The transformation that moves $t 1^{\prime}$ to $t 2^{\prime}$ is a rotation of centre $B$. So, even the point $B$, translated of $A$, is a centre of rotation of angle $\alpha$.

Proof of the second lemma (Figure 14).

Let $A$ be a centre of rotation of an angle $\alpha$ and $u$ a translation vector. The triangle $t 1$ is translated in $t 1$ ' and rotated in $t 2 . t 1$ ' is rotated about $A$ in $t 1$ '. The transformation that moves $t 2$ to $t 1$ " is a translation along the vector $u$ ', rotated of $u$ by the same angle $\alpha$ (I present here, as in other circumstances, not really a rigorous mathematical demonstration but its idea graphically illustrated, since I've thought to facilitate the comprehension of the students in this way. It is not, in any case, difficult to progress to a rigorous Euclidean proof, but the choice depends on the typology of school in which the subject is presented).

Admissible rotations

In order to understand which are the admissible rotations we have to take into account that they must be compatible with translations. The preceding lemmas bring us to the following result: the minimal admissible rotations are those whose angle is in the form $360 \%$ for , excluding the case 
Proof (The idea of the proof comes from Hibert, 1932. Another proof that uses simple trigonometric calculations is presented in Rigault, 1976). Let $u$ be a minimal translation of the group. If $A$ is $n$-fold centre of rotation (that is, the admissible rotations about $A$ are of angles $360^{\circ} / n$, where $n$ is a natural number, then, for the second lemma, there is a translation vector $v$, rotated of $u$ by an angle $360^{\circ} / \mathrm{n}$. The transformation $w$, given by the composition of $v$ and $-u$, that brings the point $B$ to $A$ and from this to $B$ ', is a translation because it is made by the composition of two translations (the red vector in Figure 15).

As the translation $w$ cannot be smaller than $u$, we have that the rotation angle $\alpha$ must be at least $60^{\circ}$ (see Figures 15, 16).

Figure 15

Inadmissible Rotation

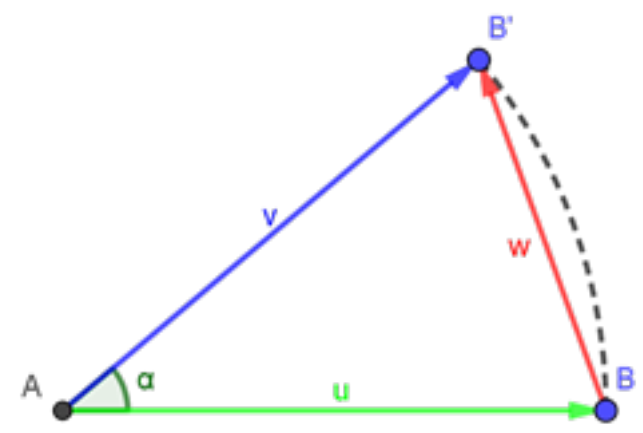

Figure 16

Admissible Rotation

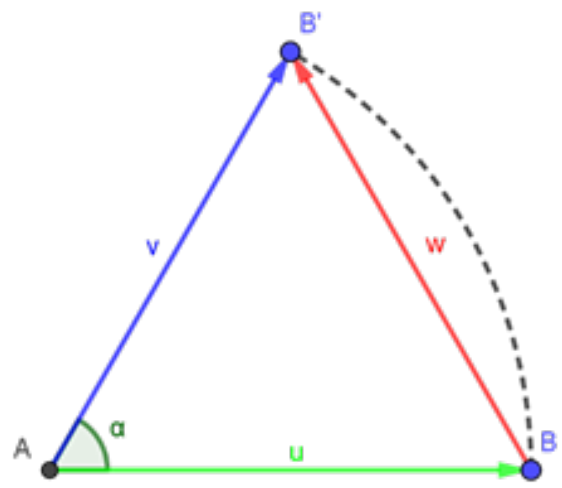

The $72^{\circ}$ rotations must be excluded because, from a $144^{\circ}$ rotation of the vector $u$ (minimal translation) a vector $v$ originates (Figure 17). The transformation $w$ that leads $A$ to $B^{\prime}$ and then to $C$ would be a translation smaller than $u$ (assumed as the minimal one).

\section{Figure 17}

Exclusion of $72^{\circ}$ Rotations

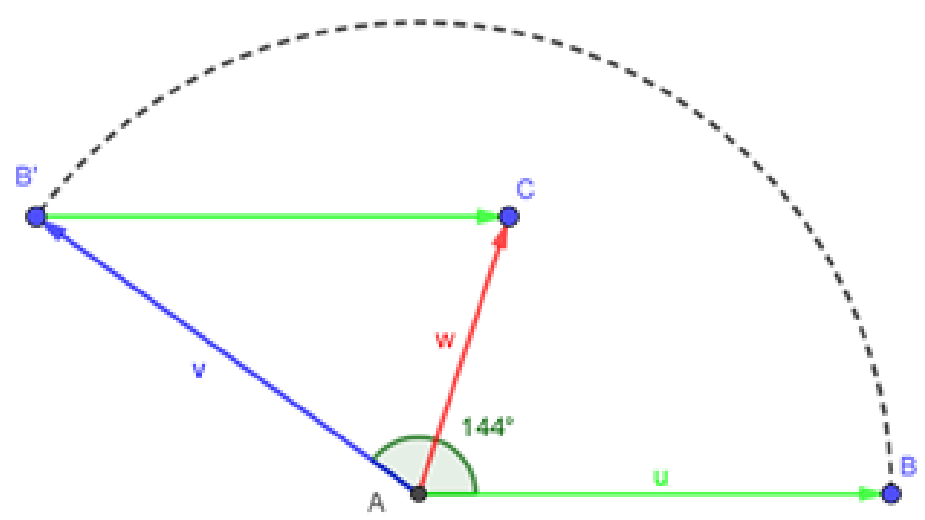

Figure 17 
Minimal rotations of $180^{\circ}$ ( $\mathrm{p} 2$ group)

Let us suppose $A$ be a 2 -fold centre of rotation and we will verify the compatibility with translations. As we have seen in the preceding lemmas, the action of translations generates a lattice of 2 -fold centres of rotation (blue points in Figure 18). The $t 1$ triangle is translated to $t 2$ by vector $v$ and the $t 2$ triangle is rotated about $B$ of $180^{\circ}$ into the $t 3$ triangle. The $t 1-t 3$ transformation is a rotation about $C$, the midpoint of $A B$ (Here, as later on, I use the property of the group of symmetries that fix a regular system of points to justify the presence of new centre of rotations).

Figure 18

The Generation of Centres of Rotation in the p 2 Group

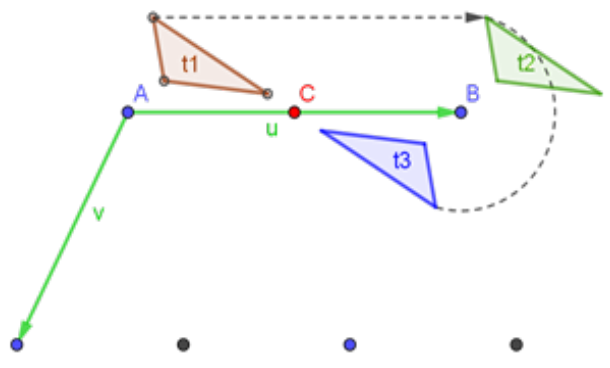

Figure 19

The Lattice of the Centres of Rotation. In Different Colours: the Different Lattices Generated by the Translation Vectors.

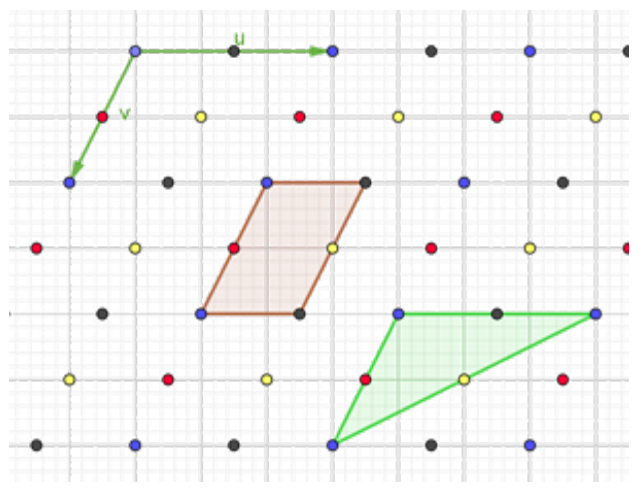

Hence, if $u$ is a translation vector that belongs to the group and it connects two 2-fold centres of rotation $A$ and $B$, then even the midpoint $C$ of $A B$ is a 2 -fold centre of rotation. In Figure 19 the lattice of the centres of rotations for this symmetry group is represented. Also depicted are some possible minimal figures (the parallelogram and the triangle), i.e. those figures which, by means of the group isometries, fill all the plane and which do not contain any points in their interior that correspond to each other by some isometry.

Possible deformations of the minimal figure

As in the preceding case, an ornamental motif inside the minimal figure can be drawn, or the figure's sides can be deformed, thus obtaining a plain tessellation. Taking a triangle as a minimal figure (shaded in Figure 20), it is possible to deform its sides, taking into account the presence of the centre of rotations in their midpoints, and the constraints they impose. One possibility is shown in Figure 20, where we can note that the three sides of the triangle are deformed independently. 


\section{Figure 20}

Figure 21

A Possible Deformation of a Triangular An Exercise on the 22 Group Minimal Figure in the p2 Group
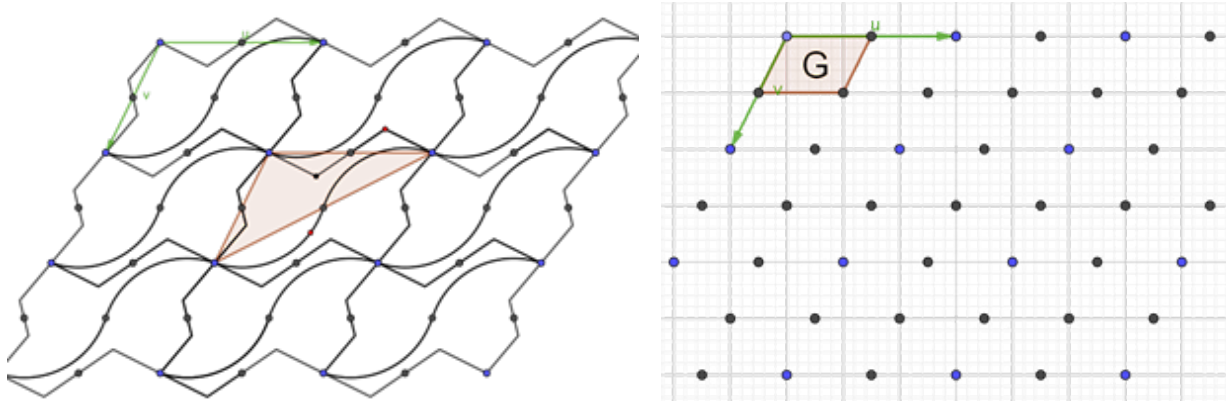

Exercise (Figure 21). Apply to the letter " $G$ " all the isometries that characterize the p2 group (rotations of $180^{\circ}$ about the points shown in the figure, translations of vectors $u$, $v$ and their compositions). Is it thus possible to complete the scheme? What can you deduce by that?

Exercise. From Escher's drawing in Figure 22, find the minimal figure, the centre of rotations and the basic parallelogram. Try to recreate the composition by tracing on a transparent paper sheet the three polygonal paths that make part of the edge of the figure as it is schematically shown in Figure 23 and then apply the rotations.

Figure 22

A p2 drawing by Escher

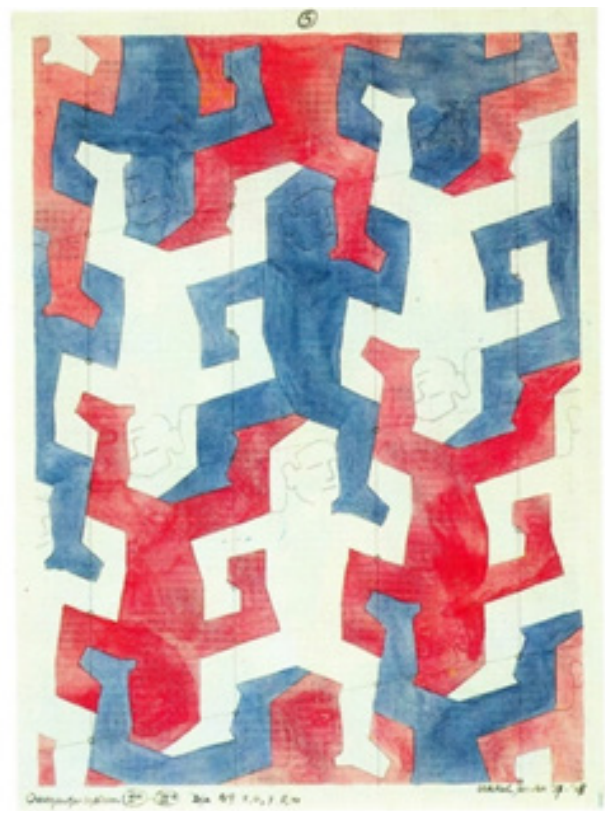

Figure 23

Reconstruction of Escher's drawing

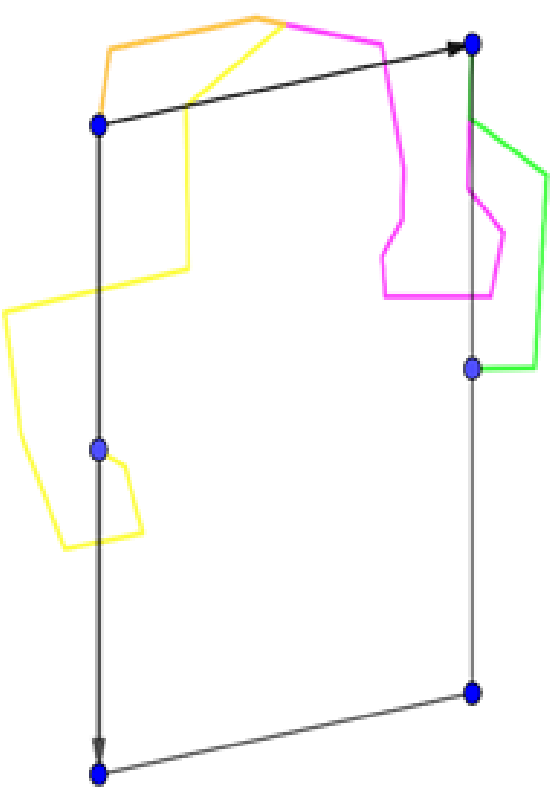

Minimal rotations of $90^{\circ}(\mathrm{p} 4$ group) 


\section{Figure 24}

Generation of the Square Lattice

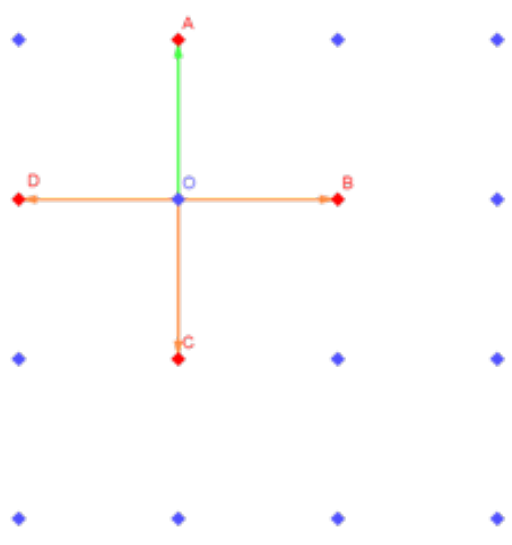

In this case (Figure 24) we assume $O$ to be a 4-fold centre of rotation. For the preceding lemmas, given the translation vector $O A$ for example, $A$ will be a 4 -fold centre of rotation and $O A$ generates, by rotation, the $O B, O C, O D$, vectors. By consequence, even the points $B, C, D$, will be 4 -fold centres of rotation.

Since every vertex of the squares that constitute the lattice is a 4-fold centre of rotation, all midpoints of the squares' sides are also centre of rotations, some of $90^{\circ}$ and some of $180^{\circ}$. In fact, recalling what we have seen with the p2 group, between two 2 -fold centres of rotation separated by a translation vector belonging to the group, there is always another 2 -fold centre of rotation.

\section{Figure 25}

Generation of Centres of Rotation in the p4 Group

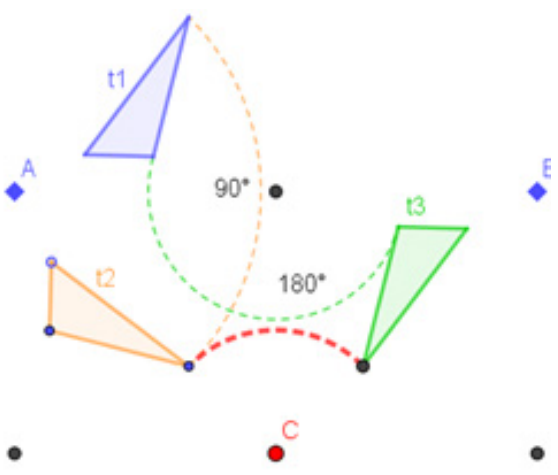


In this case, every vertex of the square cell is also a 2 -fold centre of rotation. Therefore, between two consecutive points of the lattice, there is necessarily another 2-fold centre of rotation, exactly as in the $\mathrm{p} 2$ case. This happens, for example, to the midpoint of segment $A E$, that will be a 2 -fold centre of rotation (see Figure 25).

We show now that even the centre $\mathrm{C}$ of the square cell must be a 4-fold centre of rotation. In fact, the $t 1$ triangle is moved to $t 2$ by a rotation of $90^{\circ}$ about $A$. The same triangle $t 1$ is moved to $t 3$ by a $180^{\circ}$ rotation about the midpoint of $A E$. The transformation that moves $t 2$ to $t 3$ is a rotation of $90^{\circ}$ about $C$, the centre of the square whose side is $A E$.

Figure 27 shows the lattice of the centres of rotation. We can see the minimal translation vectors and a possible minimal figure (shaded). From Figure 27 we can comprehend the group structure by watching how all the isometries of the group act on a triangle.

\section{Figure 26}

Possible Deformations of the Square Tile

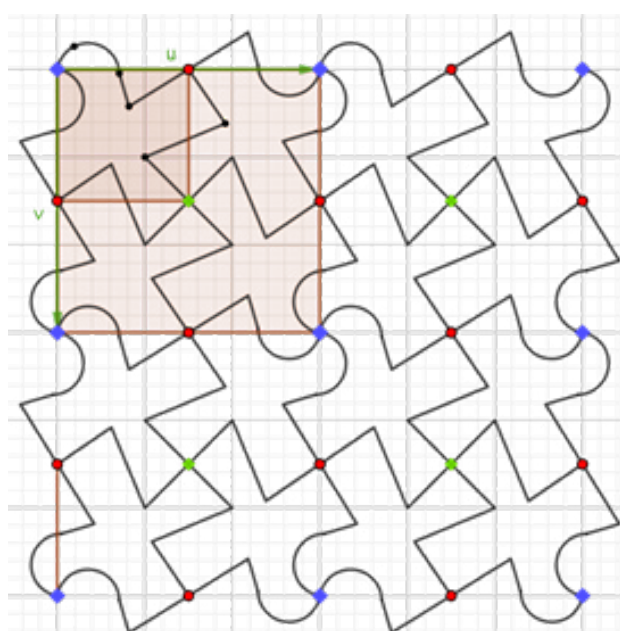

\section{Figure 27}

The Lattice of the Centres of Rotation. In Different Colours: the Lattices Generated by the Translation Vectors

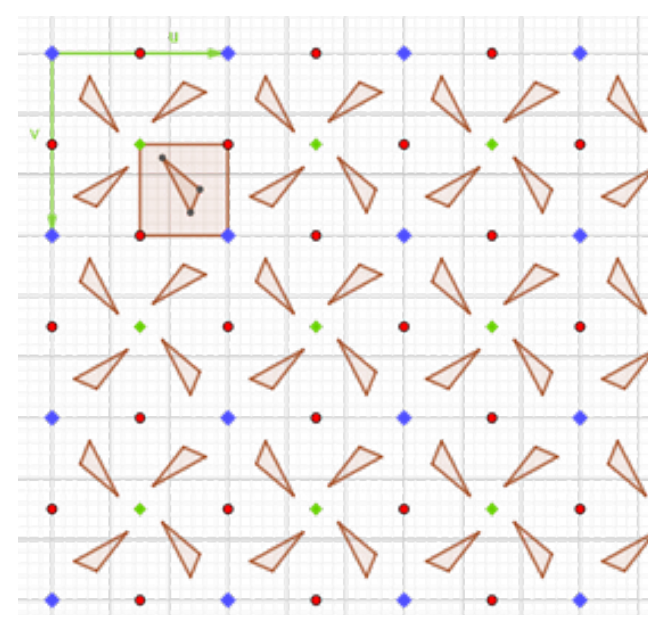

If the perimeter of the square tile is deformed to obtain a tessellation, two of the four sides of the square can be deformed independently; an example of such deformations is illustrated in Figure 26.

Exercise.

Consider Escher's fishes (Figure 28). Look for the centre of rotations and classify them; find the translation vectors and the basic parallelogram.

Verify that you can obtain the fish's form and then all the composition by the deformation of two sides of a square as is shown in Figure 29 (the two deformations are partially overlapped) and by the action of rotations. 


\section{Figure 28}

Escher's Fishes

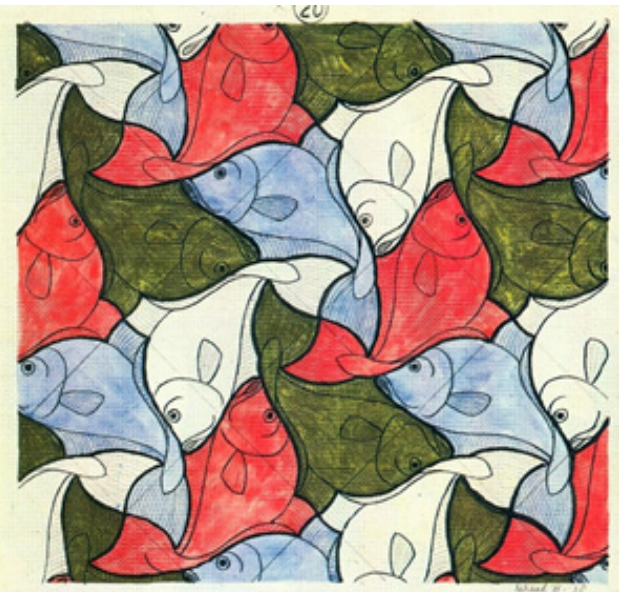

Figure 29

The Square's Sides Deformations

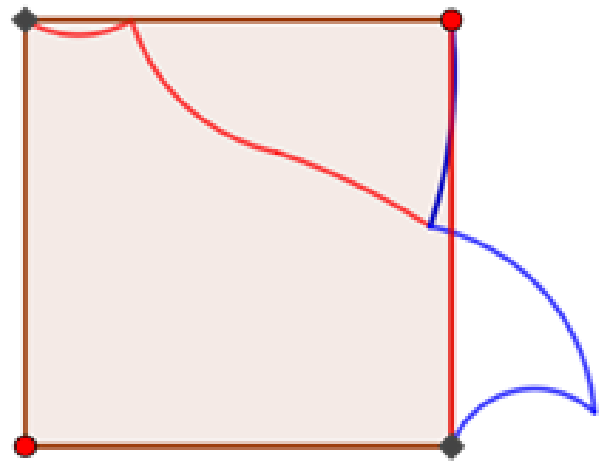

In Figure 30 we can see how a third-year high school student, in doing an assignment on the p4 group, has shown evidence of her artistic skills.

\section{Figure 30}

An "Artistic" Interpretation of 44 Group, Made by a Third-year High School Student

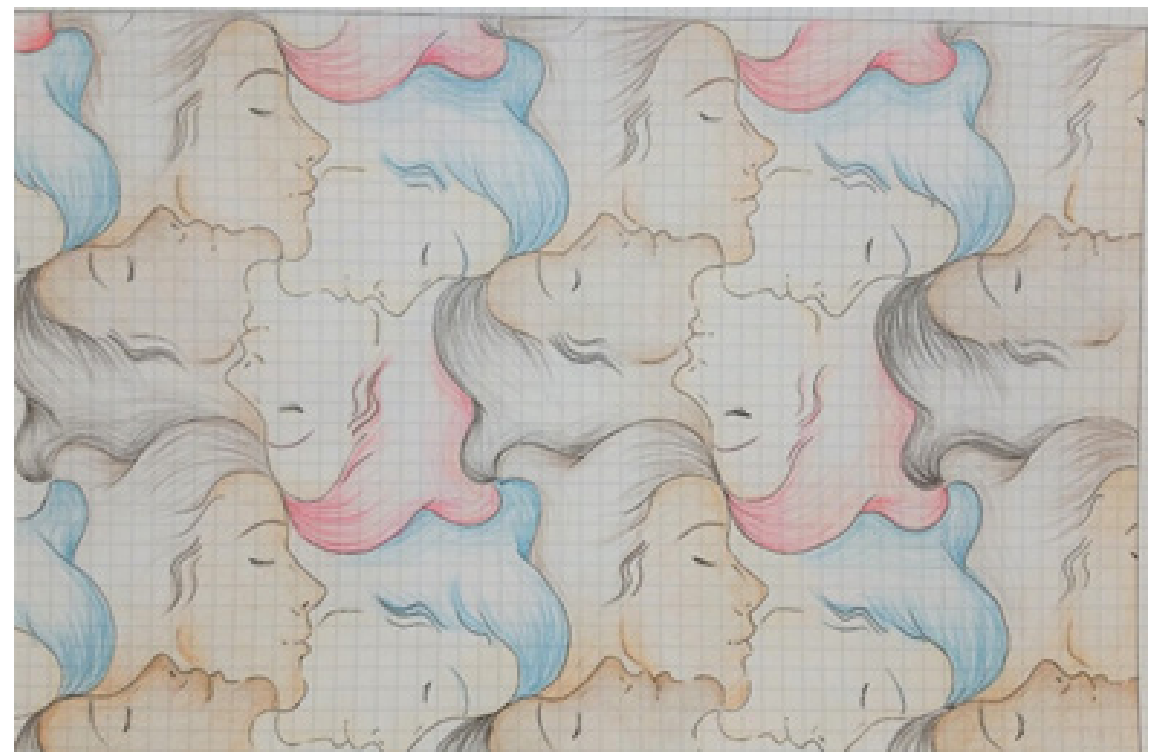

Minimal rotation of $120^{\circ}$ (p3 group)

In this case, the existence of a 3 -fold centre of rotation (that is a centre which corresponds to a minimal rotation angle of $120^{\circ}$ ) is supposed. Let $A$ be such a centre of 
rotation and $u$ a translation vector (see Figure 31), then there will be other translation vectors (as $v$ and $w$ ). Moreover, by the action of the translation vectors, every lattice point is a 3 -fold centre of rotation.

Figure 31

Generation of the Triangular Lattice

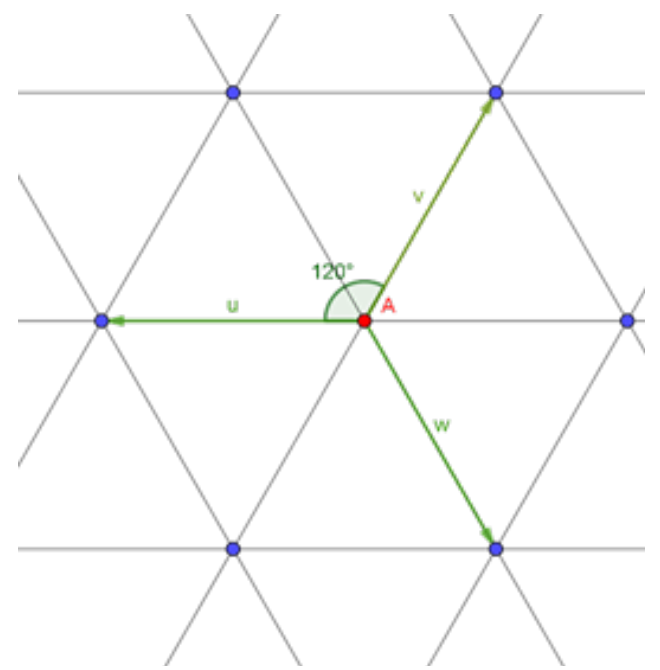

\section{Figure 32}

Generation of Centres of Rotation in the p3 Group

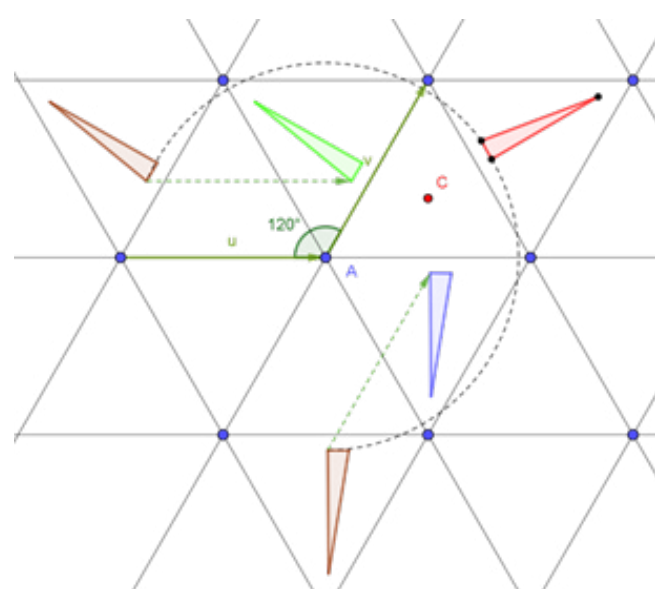

Even in this case, as in the preceding one, there are other centre of rotations. These centres will be the centres of the equilateral triangles whose vertices correspond to the lattice points represented in the figure above (see Figure 32).

The point $C$, which is the centre of an equilateral triangle, is a 3 -fold centre of rotation. In fact: the red triangle is moved into the green triangle by the consecutive action of a counter clockwise rotation about $A$ and a translation; it is also rotated into the blue triangle by the consecutive action of a clockwise rotation about $A$ and a translation. We see that the red, green and blue triangles are the transformed versions of one another, through a rotation about $C$. Therefore, all the centres of the equilateral triangles are 3 -fold centres of rotation.

In Figure 33 the $\mathrm{p} 3$ group structure is shown. There is the basic parallelogram $A C E D$ and the minimal figure $G H I L$. By the rotation of the minimal figure about $G$ we obtain a basic figure (of 3 colours) that can tile the plain by means of translations only. 


\section{Figure 33}

The p3 Group Structure

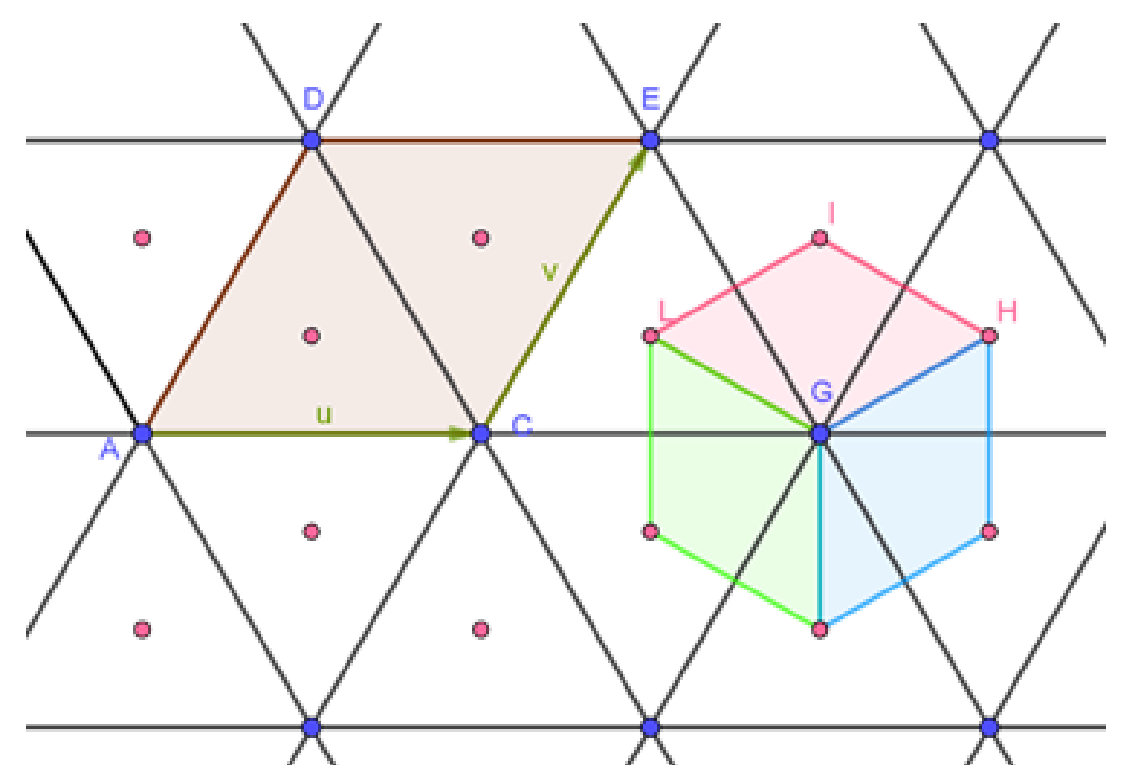

In Figure 33 there are, marked with two different colours, the initial lattice points (blue) and those points which were derived later by the composition of isometries (red).

\section{Figure 34}

Possible Deformations of the Rhombic Minimal Figure

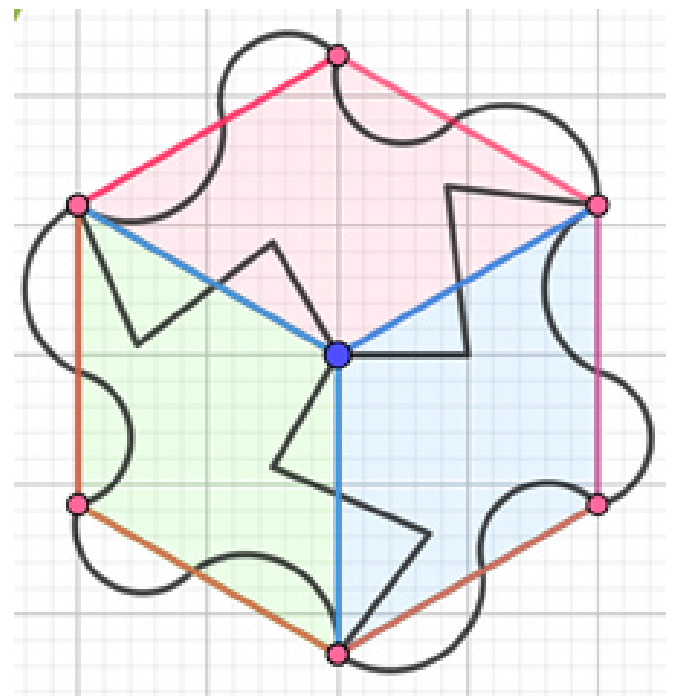

By the possible transformations of the minimal figure (rhombus with $60^{\circ}$ and $120^{\circ}$ internal angles), figures such as those in Figure 34 can be obtained, in which two sides of the rhombus are deformed independently, while the other two are obtained by rotation. 
An idea of the resulting composition is given in Figure 35:

\section{Figure 35}

A p3 Tessellation

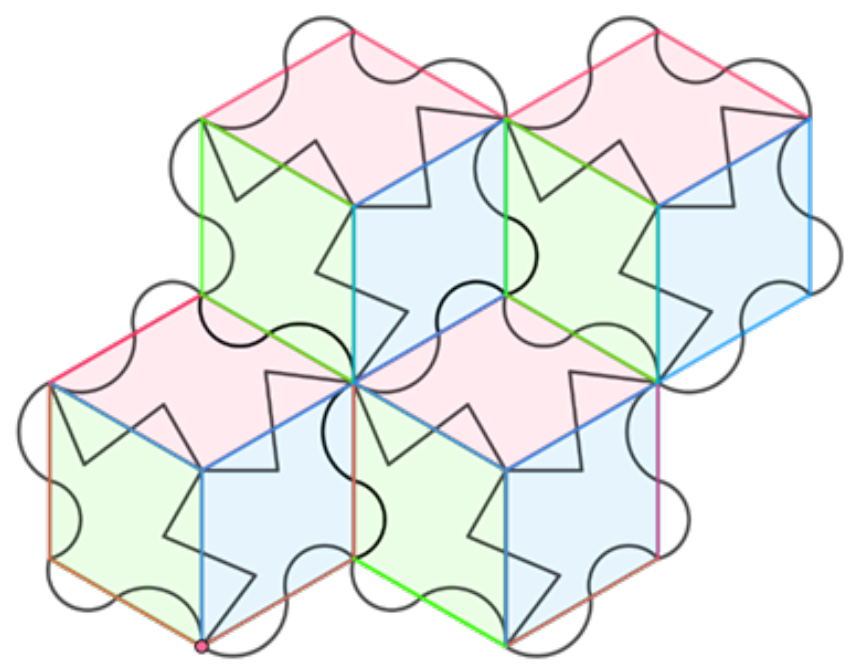

\section{Figure 36}

p3 Group Ornamental Motifs, 3rd Year High School Student

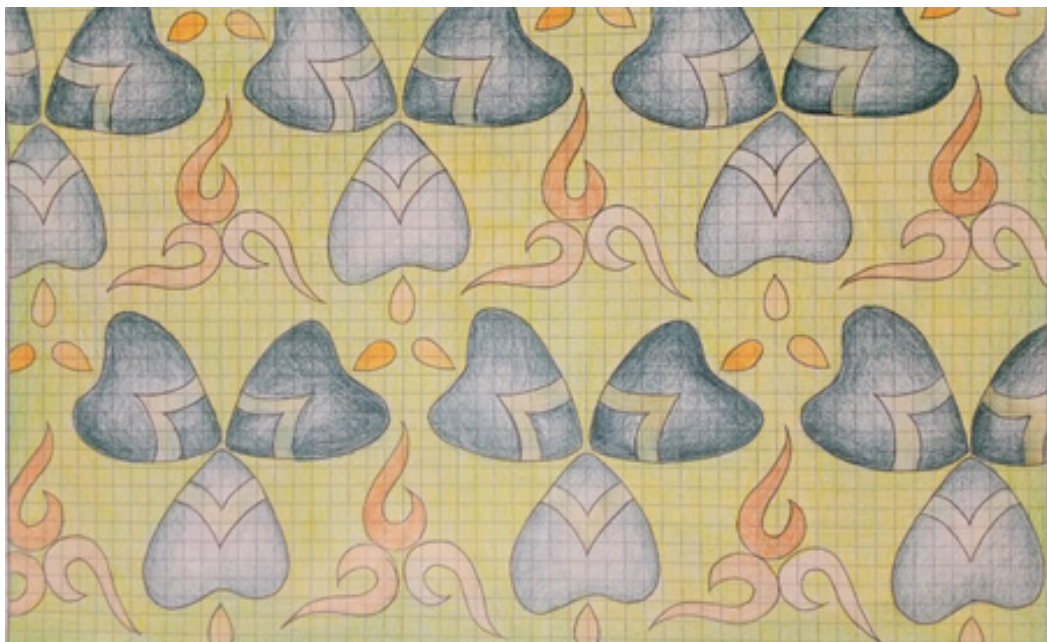

The reflection axes (pm group)

The majority of drawings and decorations observable in reality also present a reflection axes group. These reflections have to be compatible with the other isometries in the group (translations and, possibly, rotations). 
The simplest case is that one in which the symmetry group contains only axial symmetries and translations. This group is called $\mathrm{pm}$. Thus, the presence of a symmetry axis $m$, perpendicular to one of the translation vectors, is requested, while the other translation direction is parallel to the reflection axis. In this way a $m$ ' symmetry axis, translated of $m$, is created. In fact, observing Figure 37, the $t 1$ triangle is reflected in $t 2$ and translated in $t 3$, $t 2$ is translated in $t 4$ and the transformation that brings $t 3$ to $t 4$ is the reflection with axis $m$ '.

\section{Figure 37}

The Generation of Reflection Axes

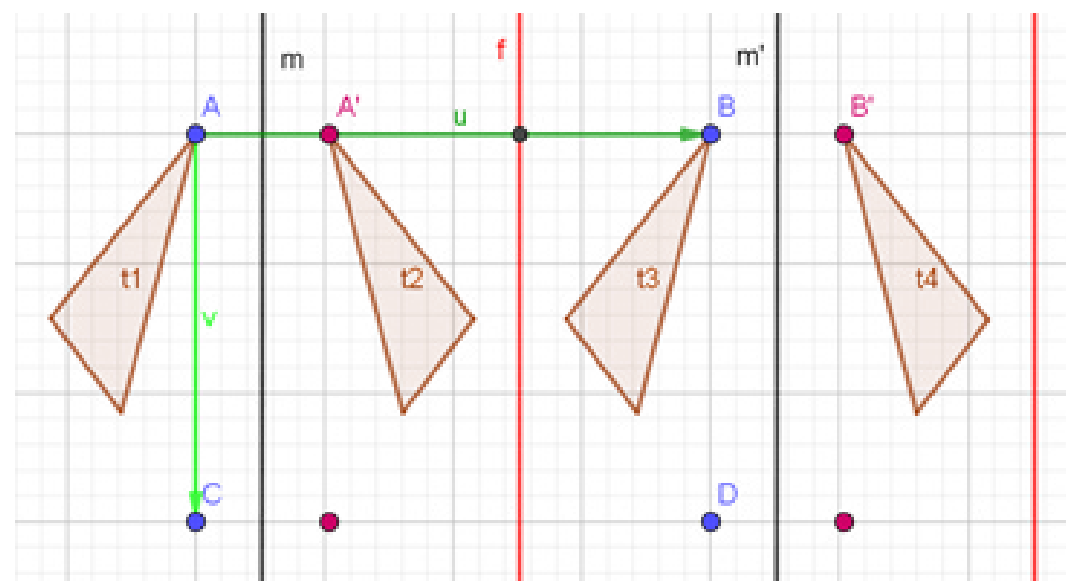

However, there are not only symmetry axes which are separated by a translation vector or by its multiples. In fact, the presence of two axes $m$ and $m$ ', separated by a translation vector $u$, produces another symmetry axis $f$ halfway between the preceding axes.

In order to prove this, let us consider the triangle $t 1$ : it is translated in $t 3$ and reflected in $t 2$. The transformation that moves $t 2$ to $t 3$ is a reflection of axis $f$ that is halfway between $m$ and $m$ ', separated by vector $\mathrm{u}$. Hence, we have infinite parallel symmetry axes separated by the $u / 2$ distance.

The presence of vertical symmetry axes does not allow deformations of the minimal rectangle's vertical sides (the rectangle is shaded in Figure 39). However, some ornaments can be drawn inside the rectangle, as in Figure 39. The only admissible deformations are those on the horizontal sides of the rectangle. 
Figure 38

A Gate, pm Group

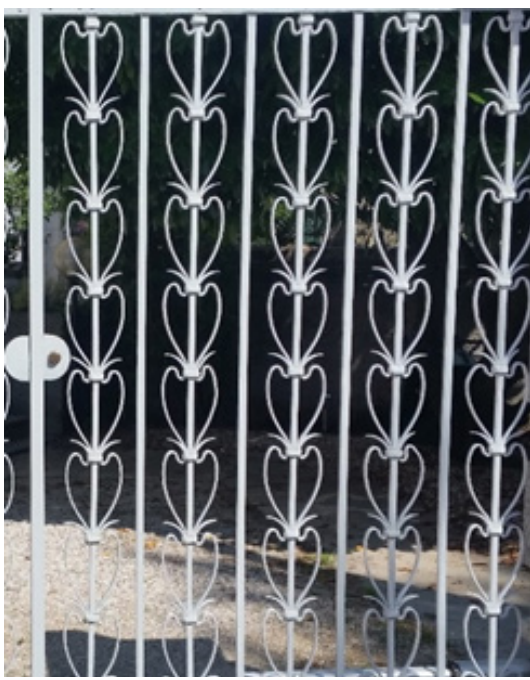

Figure 39

Possible Ornaments, pm Group

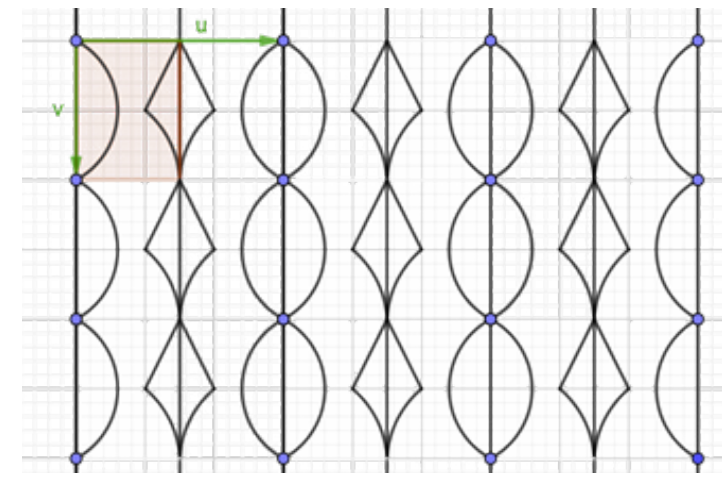

$90^{\circ}$ minimal rotations and reflection axes (p4m group)

In addition to the centres of rotation, a reflection axis can be requested in the $\mathrm{p} 4$ group, resulting in the $\mathrm{p} 4 \mathrm{~m}$ group. Such an axis should pass, for symmetry reasons, through the lattice points which correspond to 4 -fold centres of rotation $\left(90^{\circ}\right.$ minimal rotation), as the point $A$ in Figure 40. So, infinite other orthogonal reflection axes originate, separated by half of a translation vector, as we saw for the pm group

Let us see what happens with the composition of rotations and reflections. The presence of a reflection axis $f$ passing through the centre of rotation $A$ of minimal angle, causes the presence of the $g$ axis. In fact, the triangle $t 1$ is rotated in $t 2$ and $t 1$ ' in $t 2$ '. The transformation that moves $t 2$ in $t 2$ ' is the reflection of axis $g$. In the same manner we could demonstrate the presence of the $h$ reflection axis, where $h$ is the bisector of the angle between $f$ and $g$ (see Figure 40).

As a consequence of this we have that, if a n-fold centre of rotation is traversed by a reflection axis, it is automatically traversed by $n$ reflection axes. For example, for $n=4,4$ reflection axes originate through the centre of rotation, as it is shown in Figure 41. 


\section{Figure 40}

Composition of Rotations and Reflections in the General Case

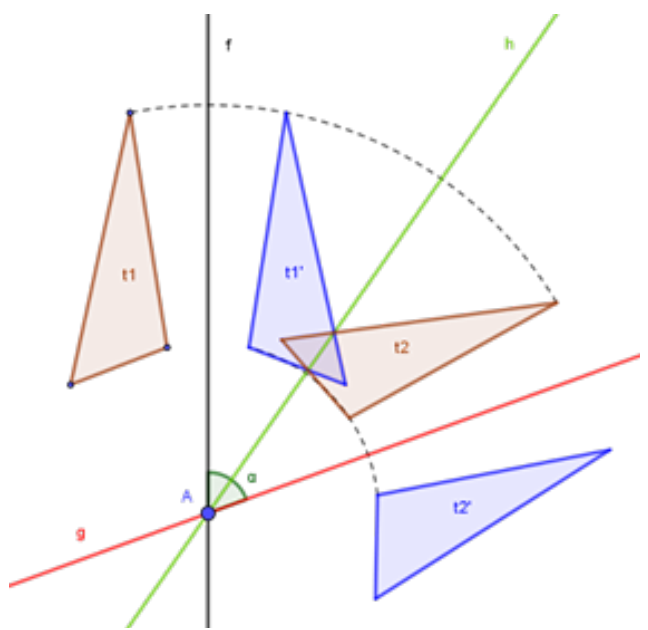

\section{Figure 41}

Composition of Rotations and Reflections in the $p 4 m$ Case

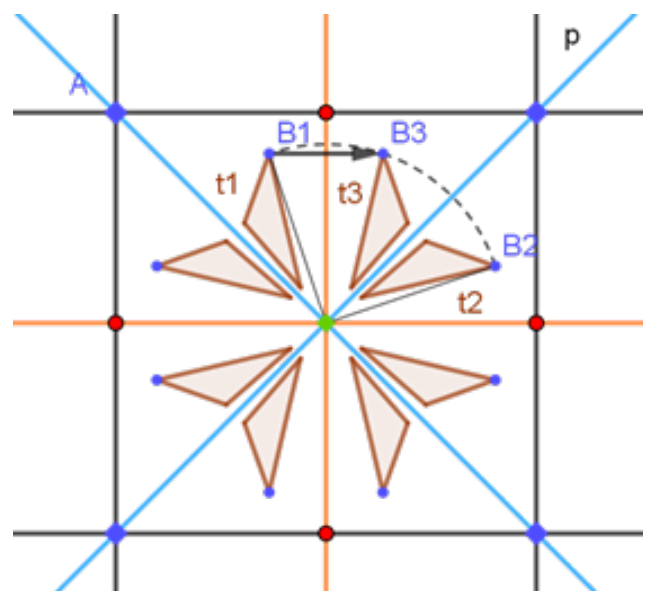

The $\mathrm{p} 4 \mathrm{~m}$ group structure is rather rigid. The presence of many reflection axes induces a strong symmetry.

\section{Figure 42}

The $44 m$ Group: the Minimal tile and its Transformations

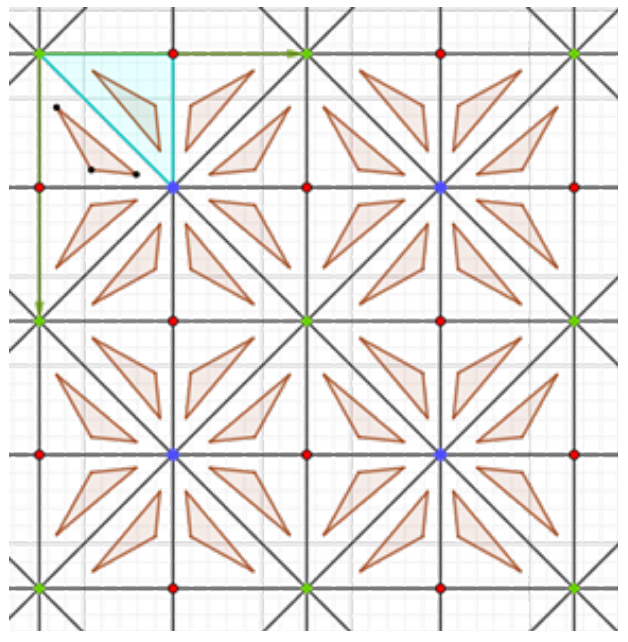

\section{Figure 43}

Ornamental Motifs in the p4m Group

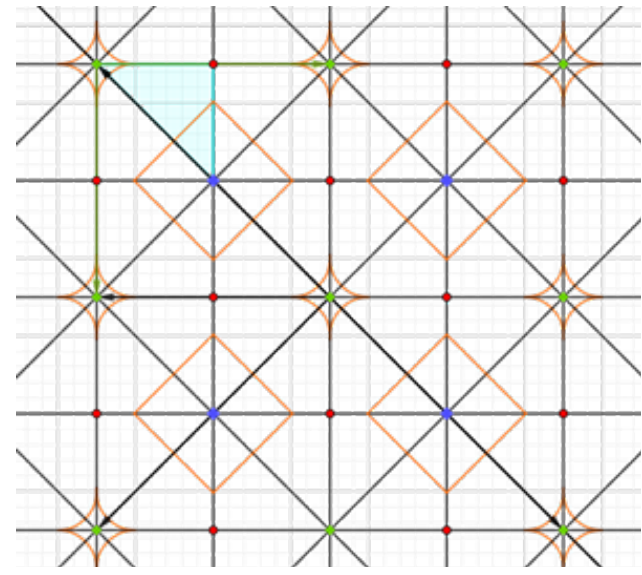

In Figure 42, a triangle is depicted and all the transformed triangles by the group isometries. The minimal tile is highlighted in light blue. The green translation vectors correspond to the sides of the basic parallelogram. The perimeter of the minimal figure cannot be deformed, but some ornamental motif can be drawn inside it. An example is given in Figure 43. 
There are many examples of compositions taken from reality which have this kind of symmetry. Some of them are shown here below.

Exercise. Analyse the following structures taken from reality. Find the minimal figure, the basic parallelogram, the centre of rotations and their nature, and the symmetry axes.

Figure 44

A Perimeter Wall

\section{Figure 45}

A Gate
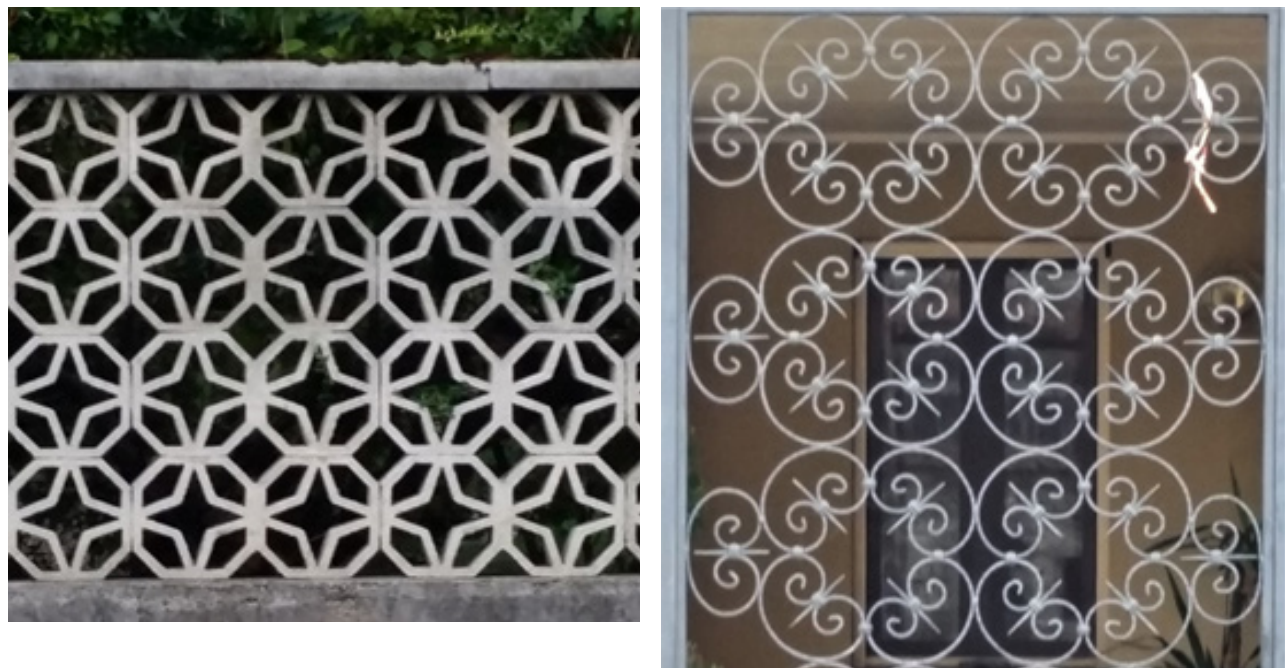

The presence of glide reflection axes ( $\mathrm{p} 4 \mathrm{~g}$ group)

In the preceding group $(\mathrm{p} 4 \mathrm{~m})$ there were 4 reflection axes for each 4-fold centre of rotation and 2 reflection axes for each 2 -fold centre of rotation.

The $\mathrm{p} 4 \mathrm{~g}$ group originates instead by the presence, inside the $\mathrm{p} 4$ group, of reflection axes passing through the 2-fold centres of rotation only. The 4-fold centres of rotation are not traversed by reflection axes. The letter "g" denotes that there are also glide reflection axes (dashed in Figure 47).

Exercise. Find, in Escher's drawing from Figure 46, the nature and the position of centre of rotations; look for the minimal figure, the translation vectors and the basic parallelogram; try to reconstruct the minimal figure, starting from the triangular tile from which it is obtained. 


\section{Figure 46}

A p4g drawing by Escher

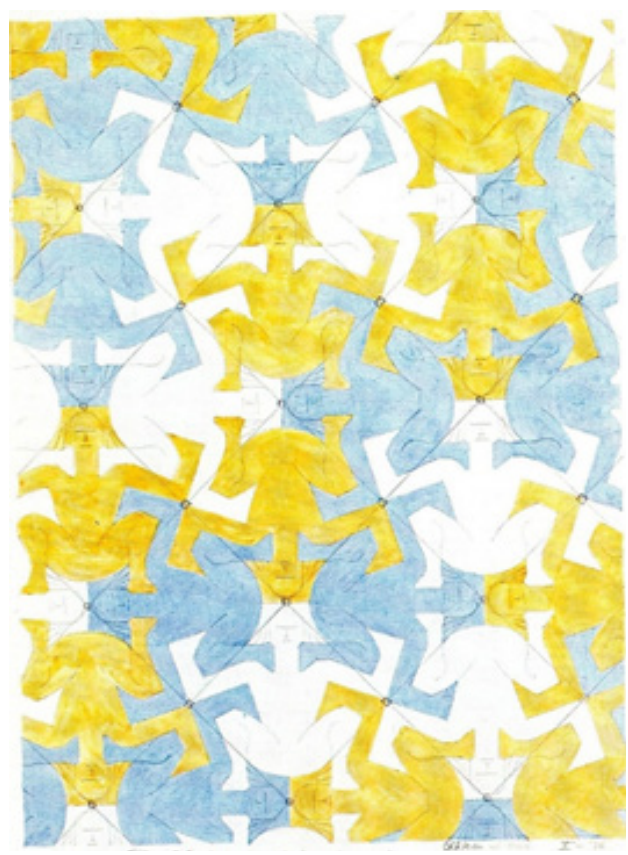

\section{Figure 47}

The $4 \mathrm{~g}$ structure

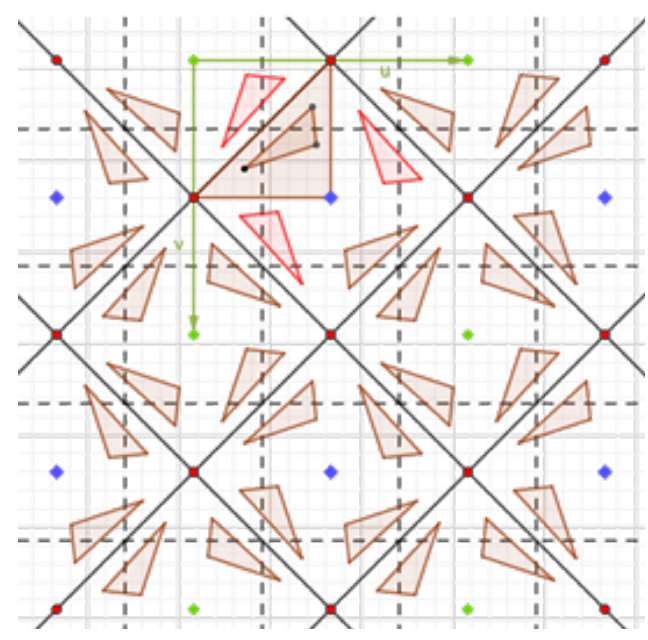

\section{Conclusions and Implications}

This activity has been positively accepted by students and they were engaged in exploring mathematics applied to their artistic and creative interests. Given the conspicuous amount of material about this subject, only some among the 17 symmetry groups were considered; anyway, this itinerary has been sufficient to provide students with the idea of how to proceed to analyse and create tessellations autonomously, even if, for lack of time, they were not led in the creation of their own compositions.

Many ideas were exchanged between teachers, in particular fashion design teachers, for whom the tessellation subject is relevant, and they have shown a deep interest in this subject. Some collaborative projects applying periodic drawings were planned, but this year, unfortunately, especially because of the COVID-19 health emergency, they could not be achieved.

The periodic drawings topic should be part of a fully-fledged artistic education, and, by studying it from a mathematical and geometrical point of view, students can enhance their skills and reach a level of complexity in their artistic composition that was previously unimaginable.

Finally, some practical recommendations can be given. The first one concerns time: if 8 hours were sufficient to give the idea of the subject, it would be necessary to employ 8 more hours at least for the learners to practice on this new subject. The second one concerns software applications: all figures presented here are made by GeoGebra free application. It is particularly suitable for making periodic drawings and carry on transformation of objects. That is why, with an appropriate amount of time, it should be a good idea to introduce the students to the use of this application. 


\section{References}

Aiton, E. J., Duncan, A. M., \& Field, J. V. (1997). The Harmony ok the world by Johannes Kepler. American Philosophical Society.

Castaldi, L., \& Ghione, F. (2011). Matematica e Arte [Mathematics and art]. Springer-Italia.

Dedò, M. (1999). Forme, simmetria, topologia [Shapes, symmetry, topology]. Decibel-Zanichelli.

Di Maggio, M. (2006). Gruppi cristallografici nell'arte di M.C. Escher [Crystallographic groups in Escher's art]. Graduation Thesis, University of "Roma Tre".

Hilbert, D., \& Cohn-Vossen, S. (1952, first edition 1932). Geometry and the imagination. Chelsea Publishing.

Morandi, P. J. (2003). The classification of wallpaper patterns: From group cohomology to Escher tessellations. New Mexico State University.

Rigault, G. (1976). Introduzione alla cristallografia [Introduction of crystallography]. Libreria editrice universitaria Levrotto \& Bella.

Schattschneider, D. (1990). Visions of symmetry. Notebooks, periodic drawings, and related works of M. C. Escher. W. H. Freeman and Company.

Weyl, H. (1952). Symmetry. Princeton University Press.

Received 12 October 2020; Accepted 08 December 2020

Cite as: Canciani, S. (2020). A didactic itinerary through mathematics, science and art on Escher's periodic drawings. Gamtamokslinis ugdymas / Natural Science Education, 17(2), 100-123. https://doi.org/10.48127/gu-nse/20.17.100

\section{Simone Canciani}

Mathematics Teacher at High School of Art (Liceo artistico "G. SELLO"), Piazza Primo Maggio 12, 3310 Udine, Italy.

E-mail: simonecanc@gmail.com

Website: http://www.arteudine.edu.it/pvw/app/UDIA0001/pvw_sito.php 